\title{
Vehicular Social Networks and Vehicular Ad-hoc Networks, Applications, Modelling Tools and Challenges: A Survey
}

\author{
Iman Mohsin Hassan \\ Department of Information Systems, College of IT, \\ University of Basrah, Iraq
}

\author{
Kareem Radhi Hassan \\ Department of Computer Science, College of IT, \\ University of Basrah, Iraq
}

\begin{abstract}
Mobile Ad Hoc Networks (MANETs) is an important category of wireless networks that can be configured dynamically without the infrastructure configuration that required in other form of networks. MANETs are able to adjust and reconfigure the connected nodes on the fly according to node movement and changeable network topologies. There are many types of MANETs one of them is Vehicular Ad hoc Networks (VANETs). VANETs purpose is to connect the smart vehicles that can be exchange a huge number of data message about the status of roads, the status of environments and the status of passengers. In this study a comprehensive survey on most recent techniques and methods used to VNETs and VSNs. First of all, the Importance of VSNs and its services are summarized. Consequently, a resent challenges in VNs and SVNs are presented, followed by the study for the most existed works that related to the VANETs and VSNs with the most important characteristics that have compared from many aspects are studied and discussed in detail. Finally, our paper presents the tools and simulation programs used to implements and developments them.
\end{abstract}

\section{General Terms}

Mobile Ad Hoc Networks (MANETs), Vehicular Social Networks (VSNs), Vehicular Ad hoc Networks (VANETs), Internet of Vehicles (IoV)

\section{Keywords}

Mobile Ad Hoc Networks (MANETs), Vehicular Social Networks (VSNs), Vehicular Ad hoc Networks (VANETs), Internet of Vehicles (IoV)

\section{INTRODUCTION}

Recently, Internet of Vehicles (IoVs) have had a positive effect on the transportation systems and Vehicular Ad hoc Networks (VANETs) the same. Intelligence of vehicles helps in developing a smart environment to share information and to develop safe transportation systems. The vehicles that wirelessly communicate with each other form the main components of this environment [1]. Although IoVs and VANETs are associated terms, the main difference between them is using the Internet services. Indeed, IoV uses the Internet services to communicate with each other. It is a big umbrella of VANET where global ID is required in IoV whereas VANET is a local network [2,3]. As a result, it can be regarded that IoV is online vehicular network while VANET is offline vehicular network as long as it is not using the Internet services. There are three communication scenarios through which vehicles can communicate with each other vehicles with vehicles V2V, vehicles with Infrastructures V2I and I2I. However, vehicles suffers from some constrains. Due to high mobility and unpredictable behavior the vehicular systems can be considered as complex systems [4]. Therefore, a realistic and efficient model needs to be think carefully about to minimize the non-realism. To achieve somewhat of those goals by using distinct, approaches. The hard defiance is how to efficiently model mobility of vehicular networks because of their constrains. Some efficiencies could be obtained by applied each strategy and this depend on the application for which the VANET is designed. [5]. on the other hand, Social Networks (SNs) are complex systems. Many social objects such as people or organizations have been communicating to not only still in contact with each other, but to also share their experiences. Relationships are keys that distinguish the human life. SNs can be classified into two fundamental kinds. These are offline social networks and online social networks [6]. It has been argued that $\mathrm{SN}$ is collected information of social objects. Offline SNs are more difficult to deal with from online SNs in term of collecting data. For this reason the most studies of the offline SNs are limited to few individuals. In contrast, data of the online SNs are mostly available. It is not required to ask individuals filling survey forms [7].

This Research is organized in six partitions with introduction. The nature and the aims of this survey is to be clearly defined in next section. Third section described the most recent challenges of this new research direction, the literature review is mentioned in the fourth section. The simulation tools that have been used in most studies are highlighted in the fifth section. The conclusion of this research will be concluded in the last section.

\section{APPLICATIONS OF VSNS}

The main possible applications of VSNs can be showed in Figure (1.1). Many novel VSNs' applications can be added to the VANET's applications. They are ranging from (safety comfort) applications. Furthermore, recommendation systems to advise options for vehicles or drivers who need them can be a wide research space.

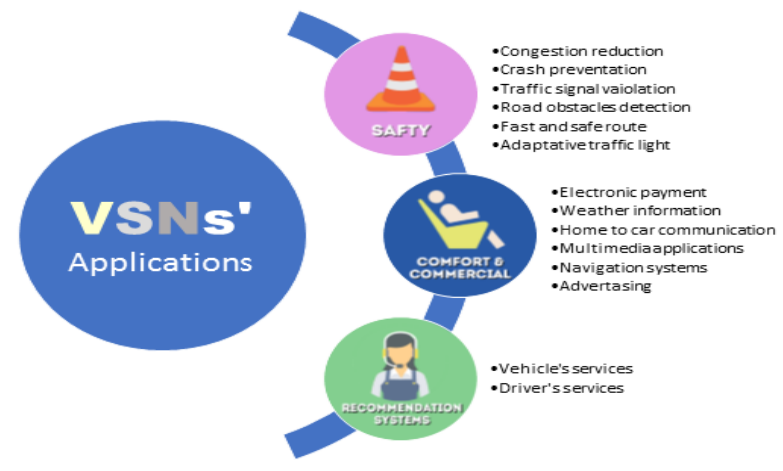

Figure 1.1 Applications of VSNs 


\section{CHALLENGES IN VNS AND VSNS}

Numerous papers have argued that there are several challenges reported in VNs [8-10]. On the other side, as VSN is a recent research direction its challenges need to be investigated. Figure 1.2 summarizes the most of these challenges including grouping $[11,12]$, Security and privacy [8, 10, 13], Mobility [2]. Information diffusion and Trust management [14]. As a result, Figure (1.2) summarizes the most challenges in both VNs and VSNs.$$
\text { Challenges }
$$

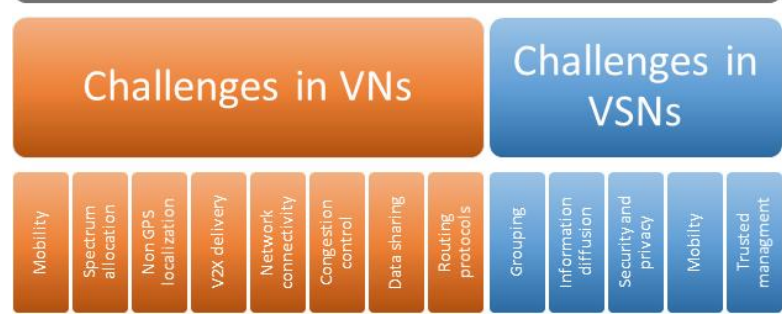

Figure 1.2 Challenges in VNs and VSNs

\section{LITERATURE REVIEW}

\subsection{Modeling Mobility and Simulation}

The simulation could be unreasonable because of models are not taking in account traffic urban scenario characteristics. There are many mobility models are convenient for traffic of vehicles simulation. [15]. some of these models either unidirectional or bidirectional simulations. The realism can be obtained by merged two simulations. A large number of simulators that have been designed for vehicular network are unidirectional [16]. Network of vehicles can be achieved by NS2 [17]. However another simulation can be used to achieve traffic simulation .for example GloMoSim is designed to simulate both wired and wireless networks and also it has capability to simulate the mobility [18]. Vehicular networks simulated by VanetMobSim [19]. It exemplifies the extension of CanuMobiSim. The NetLogo is a simulator which can be utilized in conduct of vehicles simulation [20]. A road model have been discussed using NetLogo[21]. Moreover, two simulations combined to form coupled simulations (bidirectional) First one is to simulate road traffic while second one is to simulate the network. Traffic simulation Called SUMO have been suggested in [22]. It is used to simulate the network so as to it coupled with NS-3. In [23], authors used VanetMobSim simulation to simulate the road traffic while NS-2 just as a network simulation. VISSIM is stand for "Verkehr In Städten - SIMulationsmodell" which is a German sentence (that means "Traffic in cities - simulation model"). its used to design realistic simulation for VANETs when it is coupled with NS-2 [24].

\section{a. Unidirectional VANET Simulation}

There are many mobility models are convenient for traffic of vehicles simulation. Paramics, VISSIM, SUMO, and also AIMSUM are road traffic samples. Whereas, no one from above simulator can be used to simulate the VANETs communication. Then as well, Communication Network Simulation (CNS) tools (eg. NS2, OMNeT++, Qualnet etc) are available. Interactions between vehicles conduct and vehicles communication is not easy so they are not capable of reflect this interactions [24]. Therefore a link between TFS and CNS is required and it should be interactive. Two methods to accomplish this task are available. First one is that the network simulation is fed with the output of road traffic simulation; while making both simulations to work in parallel is the second way. The last approach is appeared to be more realistic as a result of the living interactions. The big challenge is modeling the mobility of vehicles. The real movement trace model that is used for vehicles is not easy to achieve. Despite it is potential to get an estimation to this model from real world, the remaining VANETs is not easy to be simulated [15].It is may be not reasonable for all tasks the process of altering only one parameter and keeping others. In this context, researchers do a hard work to design simulations for realistic road traffic and this simulations take vehicles behaviors into consideration [15]. This simulations may be subdivided into three kinds (macroscopic, mesoscopic and microscopic) due to the granularity such that road traffic would have to be simulated [15]. Microscopic can simulate one vehicle behavior, but the first two kinds cannot do that and also their interactions with others. This model which is widespread exploited in road traffic simulations is called SUMO. It have the ability to send many commands to OMNET++ module and also sent vehicles' locations. The last one can respond to produce new nodes easily, to remove nodes when they reached their destinations furthermore nodes movement is related to their interactions in SUMO. In perspective of the network simulation, it has been recommended to simulate the network configurations before deploying nodes in real world. Actually, the simulations can be used as a result of the cost and risky environments. Moreover, it is more feasible to assess the behavior related to new protocols before actual deployment. Network communications can be simulated with many network simulations. Generally discrete event simulations are used to test the network protocols. An example of them (NS2, OMNET++, Jist etc.) such that these types are open source and also many of them are commercial such OPNET. OMNET++ and its VEINS framework will be utilized in our work.

\section{b. Bi-directionally Coupled Simulations}

Every event in VANET need to communicate with road traffic simulation and network simulation, for example waiting time for cars, information on collisions or information on obstacles. For this reason simulations were built bi-directionally coupled. Two tasks can run simultaneously. First one, in road traffic simulation will construct a node corresponding to each vehicle. While second one is related to the simulation of road traffic, where the simulation data of the network represent its output. These simulations exchange velocity or vehicle position between them.

\subsection{Linking VNs and SNs}

This dissertation does not claim that it is the first that combines techniques of social networks and techniques of vehicular networks. Nevertheless, it identifies the terms of offline VSN and online VSN. Besides, grouping vehicles in formal and casual groups is another contribution. It considers vehicles as social objects to exchange their experiences. Like humans who create social groups, vehicles can create their groups according to their own interests. The research area of bridge SNs and VNs area discover by few papers. In [25] present the concepts of bridging SNs and VNs. In Maglaras, L.A. and et al in 2016 the security side of the new multidisciplinary research direction are discussed. [14] New novel voting scheme is suggests to oversampling problems and overcome the cascading. Due these issues are widely arisen in SNs, So SNs techniques are benefit on solving these issues by giving a vote for each vehicle as how it is close to 
event. Researchers in [26] create new method for video social network and it will be used over VANET. The passengers can share the video during traveling. In [27] also develop a system which the passengers can join their friends during traveling. Finally, in [28] author proposed a study in wireless sensor network (WSNs) and VNs to analyze SNs. According to the topology and to control it, the centrality metrics of SNs will be used in WSNs VNs. This research area should be clearly view because it's contain many challenges. They are still limited attempts in the most related works. Moreover, the new direction's land-markers need more clarifications.

\subsection{Modeling Connectivity in VNs}

Connectivity of VANET has become a hot research direction since emergence of intelligent transportation systems [29]. As it has a significant effect on sharing messages in VANET, there are several papers have investigated connectivity of VANET. In 2007, the node connectivity metrics have been presented by Ho, I. and Leung. The number of connected pairs of nodes, period of connected nodes and duration of path are the main metrics to evaluate the connectivity of node [30]. Investigating the effect of roadside units on enhancing connectivity has done in highway scenario [31]. Sou et al. have emphasized in 2011 that although there are small number of roadside units the connectivity has been improved. In [32], authors in 2015 have proposed a new clustering metrics to increase the connectivity of the entire network. Relative velocity, Average velocity difference and average distance are the new metrics have been suggested to ensure stable clusters of vehicles. Routing protocol suggested by [33] in 2015 was Dealt with the continuous connectivity. The authors have assumed that vehicles are traveling in both clockwise and inverse directions. They have focused on reducing the rate of packet loss. Another work in 2016 has been argued that the numbers of connected components and giant component have possessed a strong correlation and long communication range [29]. This work has been implemented on taxis which are provided with GPSs. Authors in 2016 have suggested a study the minimum degree of node in k-connected VANET.Studying the minimum degree of node is not easy especially in k-connected VANET and the authors proposed a framework for this study. They have claimed that it is useful for studying connectivity and performance estimation in highway scenario [34].

\subsection{Grouping Objects in SNs and VNs}

To the best of knowledge, since the arrival of smart vans with the opportunity of communicating with one another, vans have developed to be ready to become social objects. In connection with this course of research many problems have arisen. The chief task, however, is how to make the connections between vans. This section will search definitions of social network grouping and the bridging rules of SNs and VNs. A part of this segment will be to examine the relevant works that have been completed so far.

\section{a. Grouping Social Objects in SNs}

To sociology the definition of social collections is a major task. A unified social collection is a set of social items considered to be knots in the graph. Such knots are linked in bidirectional links or ends which reflect the knots relationships. Numerous researches proposed discussing subgroups within social networks. These researches builds on processes such as the centrality of the degree and heuristic methods. In addition, ordinary language processing can be used to recognize people's subgroups within social networks. Through examining the interactions, nouns and phrases can be used to discuss group characteristics [35]. Degree centrality
(DC) is commonly used for measuring the degree of each knot in SNs. Betweenness centrality (BC) is to measure the number of shortest paths a certain knot has toward others. Closeness centrality (CC) is an indicator of how long each knot has the shortest path. On the other hand, author in [36] has suggested a profit aware grouping method. It depends on skills of individuals who can cooperate to accomplish sets of tasks. This grouping team will increase the total benefit of the job which the team can complete. A new individuals group has been proposed in [11] Building on a regional user similarity that takes into account its limitations and the importance of social networks. Pedestrian classification was identified in [37], depending on the changing network of track interactions (ETIN). Pedestrian tracks are represented as knots while their actions is represented as weighted edges. Although many studies have concentrated on grouping in social networks, this is still an open, challenging research problem [35].

\section{b. Grouping Social Objects in VNs}

Nemours researchers have argued that clustering technique has positive effect on routing data in MANETs [32]. However, in VANET there is a different tale. The high mobility makes clustering technique in VANETs difficult task as it is above-mentioned. Moreover, issues of clustering in mobile systems have to be addressed. Table (1.4) shows some of the works related to dynamic clustering in VNs.

\subsection{Information Dissemination in VSNs}

The different literatures have proposed different data sharing techniques. So this section will discuss the most recent literatures in this research direction. In [38], a new simple and efficient data sharing technique is proposed depending on density of surrounding vehicles. According to that, each vehicle can use a suitable probability to rebroadcast messages to reduce the number of sent messages. In [39], authors assert that priority can be based to reduce transmitted data. They suggest a new scheduling technique depending on expiry time. Authors in [40] propos a hybrid algorithm to collect data using RFID to prevent fake messages.in [41], authors use cooperative awareness among vehicles to share traffic data According to this paper, each vehicles share its traffic data unit (TDU) with a number of received TDUs from other vehicles. Complex network theory is used to share taxi dataset [42]. In this paper, clustering is used to select the station and model is designed to select the source of information. The context of data is based to share in [43]. The cluster heads aggregate data according to the context. Cluster based semantic data aggregation (CBSDA) is proposed in [44]. The road is broken into different sections depending on cluster-ID and then data aggregated in each cluster. Cost awareness Multicasting has been suggested in [45]. In this paper, messages are delivered for a specific number of vehicles. In [46], a new multi-hop broadcast algorithm has been proposed depending on link quality of neighboring vehicles. According to the previous literatures data sharing is a recent challenge in VANETs.

Table 1.1 mobility models researches

\begin{tabular}{|c|c|c|c|c|c|c|c|c|c|}
\hline 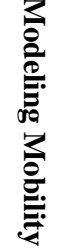 & 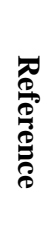 & 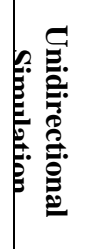 & 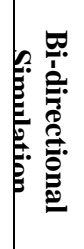 & $\begin{array}{l}Z \\
\text { N } \\
\mathbf{T} \\
\mathbf{W}\end{array}$ & $\begin{array}{l}\frac{\Omega}{0} \\
\frac{2}{8} \\
\frac{0}{0}\end{array}$ & 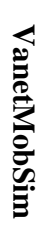 & 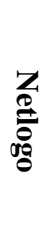 & $\frac{\pi}{5}$ & \begin{tabular}{l}
$\leq$ \\
\multirow{6}{2}{} \\
3 \\
3
\end{tabular} \\
\hline
\end{tabular}




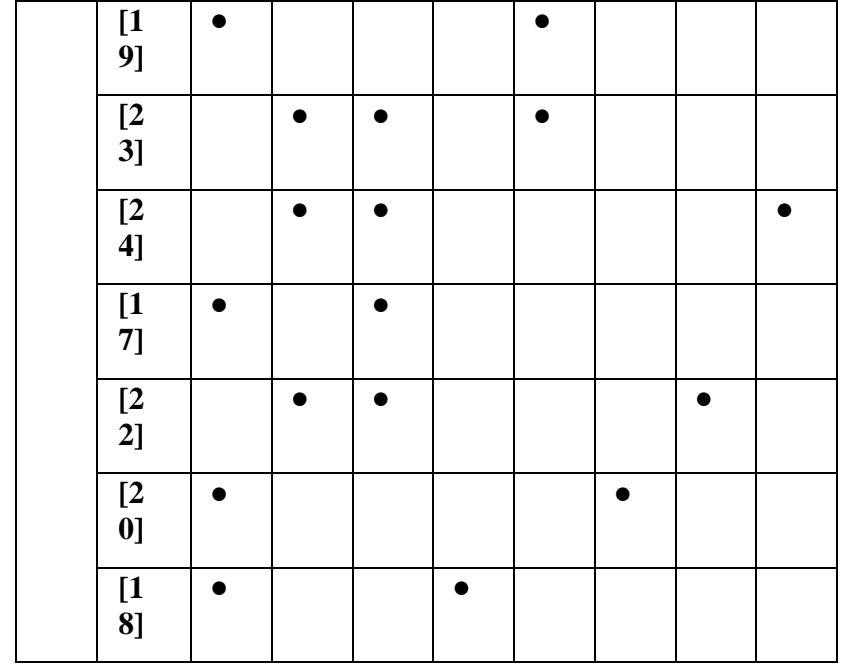

Table 1.2 VNs and SNs linking researches

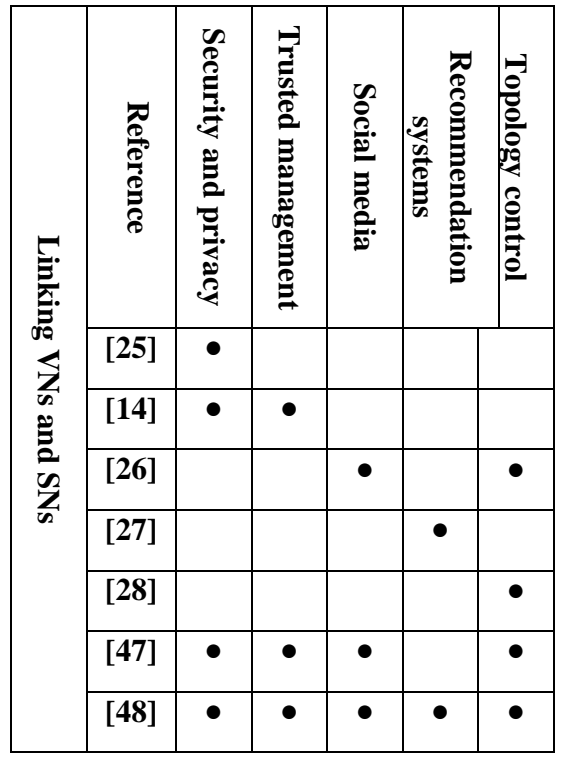

Table 1.3 VNs connectivity researches

\begin{tabular}{|c|c|c|c|c|c|c|c|}
\hline \multirow{7}{*}{ 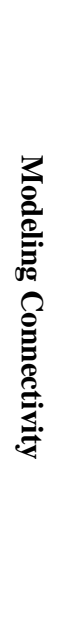 } & $\begin{array}{l}\ddot{0} \\
\stackrel{0}{0} \\
0 \\
0 \\
0\end{array}$ & $\begin{array}{l}Z \\
\vdots \\
0 \\
\frac{0}{0} \\
\frac{9}{2} \\
0\end{array}$ & $\underset{\mathscr{\sigma}}{\mathscr{E}}$ & 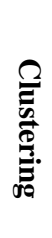 & 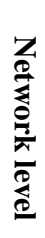 & $\begin{array}{l}\frac{Q}{0} \\
0 \\
0 \\
0 \\
0 \\
0 \\
0 \\
0\end{array}$ & 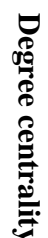 \\
\hline & {$[\mathbf{3 0}]$} & $\bullet$ & & & & & $\bullet$ \\
\hline & [31] & & • & & $\bullet$ & & \\
\hline & [32] & & & $\bullet$ & • & & \\
\hline & {$[$ [33] } & $\bullet$ & & & • & & $\bullet$ \\
\hline & [29] & & & • & & • & \\
\hline & {$[\mathbf{3 4 ]}$} & $\bullet$ & & & & & $\bullet$ \\
\hline
\end{tabular}

Table 1.4 Clustering in VNs researches

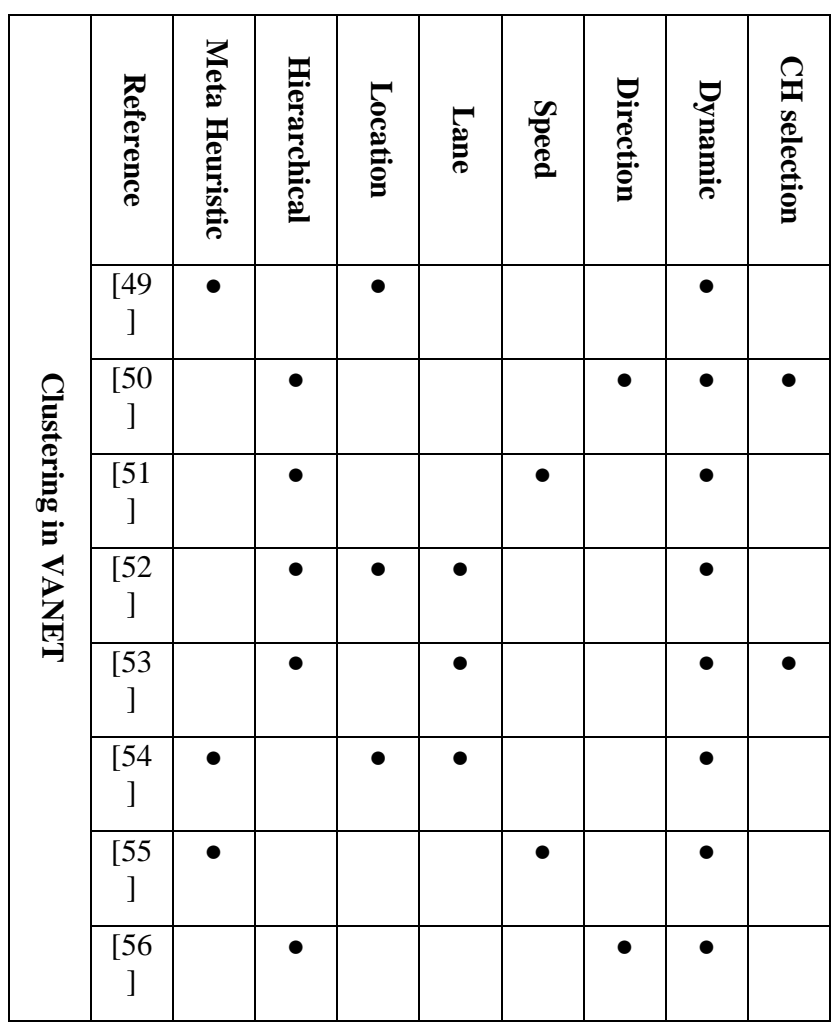

Table 1.5 information sharing in VNs researches

\begin{tabular}{|c|c|c|c|c|c|c|c|c|c|}
\hline \multirow{10}{*}{ 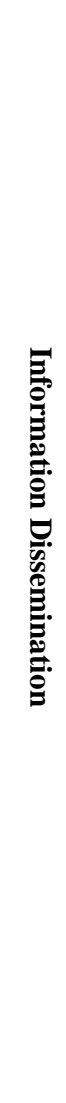 } & 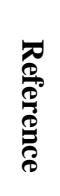 & 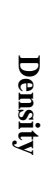 & 兄 & 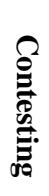 & 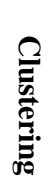 & 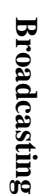 & 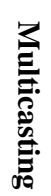 & $\underset{殳}{2}$ & న్ \\
\hline & $\begin{array}{c}{[38} \\
]\end{array}$ & $\bullet$ & & & & & & $\bullet$ & \\
\hline & $\begin{array}{c}\text { [39 } \\
]\end{array}$ & & $\bullet$ & & & & & $\bullet$ & $\bullet$ \\
\hline & $\begin{array}{c}{[40} \\
]\end{array}$ & & & & $\bullet$ & & & $\bullet$ & \\
\hline & $\begin{array}{c}{[41} \\
]\end{array}$ & $\bullet$ & & & & $\bullet$ & & $\bullet$ & \\
\hline & $\begin{array}{c}{[42} \\
]\end{array}$ & & $\bullet$ & & $\bullet$ & & & $\bullet$ & $\bullet$ \\
\hline & $\begin{array}{c}{[43} \\
]\end{array}$ & & $\bullet$ & $\bullet$ & & & $\bullet$ & $\bullet$ & $\bullet$ \\
\hline & $\begin{array}{c}{[44} \\
]\end{array}$ & & & $\bullet$ & $\bullet$ & & & $\bullet$ & \\
\hline & $\begin{array}{c}{[45} \\
]\end{array}$ & & & $\bullet$ & & & $\bullet$ & $\bullet$ & $\bullet$ \\
\hline & $\begin{array}{c}{[46} \\
]\end{array}$ & & $\bullet$ & & & $\bullet$ & & $\bullet$ & $\bullet$ \\
\hline
\end{tabular}




\section{SIMULATION TOOLS \\ 5.1. Networks Simulations}

The most important step in the WNs design is evaluating the performance of algorithms and protocols. Claiming that protocols or algorithms have a good performance is needed to evidence. Since executing suggested systems is difficult in the real world, simulators are available for this task [57]. Some of the familiar simulators will be discussed in the next paragraphs.

\section{a. Academic Simulators}

Four familiar academic simulators will be discussed in the next four paragraphs $[58,59]$.

Ns-2 and Ns-3: This simulator is built in C++ programming language. Ns-3 is extension of Ns-2, in which scripts can be written by $\mathrm{C}++$ or Python [60].

OMNeT++: it is also compatible with $\mathrm{C}++$ environment. A good infrastructure is provided to simulate WSNs. IEEE.802.11, SCSL, FDDI and TCP/IP protocols are supported by OMNet++. In addition, a good scalability is also available (number of nodes).

GLoMoSim: c-based simulator designed by parallel programming protocols of wireless networks are purely supports by GLoMoSim. It is also scalable including approximately 1000 nodes.

Ptolemy: Ptolemy is java-based simulator to support concurrent. Moreover, real time systems and heterogeneous systems are supported by Ptolemy. Nevertheless, scalability of Ptolemy is limited to few nodes.

\section{b. Commercial simulators}

QualNet: The real time networks are supported by QualNet simulator. It provides extension and interface to other simulators [59].

OPNET: This simulator is regarded as hierarchical simulator. In fact, it provides a set of processes for each node like Finite State Machine (FSM). Three links types are provided by OPNET: bus, wireless, and point-to-point [60].

\subsection{Vehicular Networks Simulations}

Due to high mobility in these networks, the simulation of realistic vehicular networks is a big challenge because of their restrictions [5]. Several simulations are obtainable to simulate the vehicular networks. Even so, the realistic is not found in all. Thus, realistic simulation is required to achieve realism. Road traffic analyzing is done by combining the road traffic simulator (SUMO) and the network simulator (VEINS) and this is an example of realistic simulation. bi-directionally coupled simulations is used to enhance vehicles behavior. The cost and the time of studying such those cases can be also decreased. Indeed, tests of real world might be hazardous, expensively and take a long time.

\section{a. Net Logo}

It is a sparkling tool that is used for agent behavior simulation. Several papers have used it to simulate different works related to vehicular networks $[20,21,61-65]$. Designing an ABM requires to define three main components agents, environment, and interactions. Actually, this process is not all. Describing agent-agent and agent-environment interactions is another need designer should carefully take in account. Besides, there are also two additional components. The first one is Observer which is responsible to ask agents what they should do. The second component is Schedule which is related to what observer should use to ask agents. In this context, Net Logo provides tow buttons in its interface SETUP and GO. The Designer clicks SETUP and then GO to schedule these actions [60].

\section{b. GraphStream}

It is a dynamic graph Java library to deal with the dynamic features of graphs [68]. The main task of GraphStream is to model the dynamic interactions of different size networks. The library includes many components to generate streams of events called SOURCES. On the other side, there are other components called SINKS that receive these events and then process them. SINKS are similar in some sense to listeners. This scenario is widely used in the Java world.

\section{CONCLUSIONS}

to make the transportation systems work as smart systems by utilize the new smart technologies and implement it in the fields of vehicular social network. In these years there are many efforts to make a hybrid systems that can be consists of social networks and vehicular networks. It is very important to study this fields and make a good survey about it to discover aims, applications, challenges to build a foundation to understand how these techniques are works and to study the most important studies and research as in table (1-4), whereas table (5) represent the comparison among the researches that works on information sharing in VNs and the most important features that focused in each research.

\section{REFERENCES}

[1] A. Botta, W. de Donato, V. Persico, and A. Pescapé, "Integration of Cloud computing and Internet of Things: A survey," Future Generation Computer Systems, vol. 56, pp. 684-700, 3// 2016.

[2] Avinash Devare, Archana Hande, Anandmohan Jha, Sandhya Sanap, and S. Gawade, "A Survey on Internet of Things for Smart Vehicles," IJRSET, vol. 5, no. 2, 2016.

[3] M. Keertikumar, M. Shubham, and R. M. Banakar, "Evolution of IoT in smart vehicles: An overview," in 2015 International Conference on Green Computing and Internet of Things (ICGCIoT), 2015, pp. 804-809.

[4] S. Hiroki, "<Introduction to the Modeling and Analysis of Complex Systems>," New York: Open SUNY Textbooks, 2015.

[5] S. Al-Sultan, M. M. Al-Doori, A. H. Al-Bayatti, and H Zedan, "A comprehensive survey on vehicular Ad Hoc network," Journal of Network and Computer Applications, vol. 37, pp. 380-392, 1// 2014.

[6] S. S. De and S. Dehuri, "Machine Learning for Auspicious Social Network Mining," in Social Networking: Springer, 2014, pp. 45-83.

[7] S. Ghosh and N. Ganguly, "Structure and evolution of online social networks," in Social Networking: Springer, 2014, pp. 23-44.

[8] T. Darwish, K. A. Bakar, and A. Hashim, "Green geographical routing in vehicular ad hoc networks: Advances and challenges," Computers \& Electrical Engineering, 2016.

[9] K. Zheng, L. Hou, H. Meng, Q. Zheng, N. Lu, and L. Lei, "Soft-defined heterogeneous vehicular network: architecture and challenges," arXiv preprint arXiv:1510.06579, 2015.

[10] K. Zheng, Q. Zheng, P. Chatzimisios, W. Xiang, and Y. Zhou, "Heterogeneous Vehicular Networking: A Survey on Architecture, Challenges, and Solutions," IEEE 
Communications Surveys \& Tutorials, vol. 17, no. 4, pp. 2377-2396, 2015.

[11] S. Alsaleh, R. Nayak, and Y. Xu, "Grouping people in social networks using a weighted multi-constraints clustering method," in Fuzzy Systems (FUZZ-IEEE), 2012 IEEE International Conference on, 2012, pp. 1-8: IEEE.

[12] A. Alsayat and H. El-Sayed, "Social media analysis using optimized KMeans clustering," in Software Engineering Research, Management and Applications (SERA), 2016 IEEE 14th International Conference on, 2016, pp. 61-66: IEEE.

[13] L. Zhang, C. Hu, Q. Wu, J. Domingo-Ferrer, and B. Qin, "Privacypreserving vehicular communication authentication with hierarchical aggregation and fast response," IEEE Transactions on Computers, vol. 65, no. 8, pp. 2562-2574, 2016.

[14] Z. Huang, S. Ruj, M. A. Cavenaghi, M. Stojmenovic, and A. Nayak, "A social network approach to trust management in VANETs," Peer-to-Peer Networking and Applications, vol. 7, no. 3, pp. 229-242, 2014.

[15] C. Sommer, R. German, and F. Dressler, "Bidirectionally coupled network and road traffic simulation for improved IVC analysis," IEEE Transactions on Mobile Computing, vol. 10, no. 1, pp. 3-15, 2011.

[16] F. J. Martinez, C. K. Toh, J. C. Cano, C. T. Calafate, and P. Manzoni, "A survey and comparative study of simulators for vehicular ad hoc networks (VANETs)," Wireless Communications and Mobile Computing, vol. 11, no. 7, pp. 813-828, 2011.

[17] N. V. Sebastian and T. Jeyaprakash, "Appraising Vehicular ADHOC Networks Routing Protocols Using NS2," 2014.

[18] A. Hassan, "VANET simulation," ed: Högskolan i Halmstad/Sektionen för Informationsvetenskap, Dataoch Elektroteknik (IDE), 2009.

[19] A. M. Alwakeel, "Implementations Of The DTM, DADCQ And SLAB VANET Broadcast Protocols For The Ns-3 Simulator," FLORIDA ATLANTIC UNIVERSITY, 2016.

[20] W. Shanshan and Z. Chunxiao, "NetLogo Based Model for VANET Behaviors Dynamic Research," in Intelligent System Design and Engineering Applications (ISDEA), 2013 Third International Conferenceon, 2013, pp. 990993: IEEE.

[21] S. T. Hasson and Z. Y. Hasan, "Simulating Road Modeling Approach's in Vanet Environment Using Net Logo," Research Journal of Applied Sciences, vol. 11, no. 10, pp. 1130-1136, 2016.

[22] Y. Su, H. Cai, and J. Shi, "An improved realistic mobility model and mechanism for VANET based on SUMO and NS3 collaborative simulations," in Parallel and Distributed Systems (ICPADS), $201420^{\text {th }}$ IEEE International Conference on, 2014, pp. 900-905: IEEE.

[23] R. C. Poonia and D. Bhargava, "A Review of Coupling Simulator for Vehicular Ad-hoc Networks," 2016.

[24] J. Sun, Y. Yang, and K. Li, "Integrated coupling of road traffic and network simulation for realistic emulation of connected vehicle applications, Simulation, vol. 92, no. 5, pp. 447-457, 2016.
[25] L. A. Maglaras, A. H. Al-Bayatti, Y. He, I. Wagner, and H. Janicke, "Social internet of vehicles for smart cities," Journal of Sensor and Actuator Networks, vol. 5, no. 1, p. 3, 2016.

[26] K. M. Alam, M. Saini, D. T. Ahmed, and A. El Saddik, "VeDi: A vehicular crowd-sourced video social network for VANETs," in Local Computer Networks Workshops (LCN Workshops), 2014 IEEE 39th Conference on, 2014, pp. 738-745: IEEE

[27] A. Elbery, M. ElNainay, F. Chen, C.-T. Lu, and J. Kendall, "A carpooling recommendation system based on social vanet and geo-social data," in Proceedings of the 21st ACM SIGSPATIAL International Conference on Advances in Geographic Information Systems, 2013, pp. 556-559: ACM.

[28] A. Papadimitriou, D. Katsaros, and Y. Manolopoulos, "Social network analysis and its applications in wireless sensor and vehicular networks," in International Conference on e-Democracy, 2009, pp. 411-420: Springer.

[29] H. Feng and Y. Xu, "An empirical study on evolution of the connectivity for VANETs based on taxi GPS traces," International Journal of Distributed Sensor Networks, 2016.

[30] I. W. H. Ho and K. K. Leung, "Node connectivity in vehicular ad hoc networks with structured mobility," in Local Computer Networks, 2007LCN 2007. 32nd IEEE Conference on, 2007, pp. 635-642: IEEE.

[31] S.-I. Sou and O. K. Tonguz, "Enhancing VANET connectivity through roadside units on highways," IEEE transactions on vehicular technology, vol. 60 , no. 8, pp. 3586-3602, 2011.

[32] M. Aissa, A. Belghith, and B. Bouhdid, "Cluster connectivity assurance metrics in vehicular ad hoc networks," Procedia Computer Science, vol. 52, pp. 294301, 2015.

[33] B. Pete and P. Jaini, "Continuous connectivity aware routing in VANET using hybrid protocol," in Electronics and Communication Systems (ICECS), 2015 2nd International Conference on, 2015, pp. 223-226: IEEE.

[34] W. Xiong, J. Xu, Y. Li, N. Zhao, X. Wan, and J. Liang, "Minimum node degree of k-connected vehicular ad hoc networks in highway scenarios," EURASIP Journal on Wireless Communications and Networking, vol. 2016, no. 1, p. 32, 2016.

[35] S. Sharma and G. Purohit, "Methods of Tracking Online Community in Social Network," in Social Networking: Springer, 2014, pp. 129-146.

[36] S. Tang, "Profit-aware Team Grouping in Social Networks: A Generalized Cover Decomposition Approach," arXiv preprint arXiv:1605.03205, 2016.

[37] L. Feng and B. Bhanu, "Understanding dynamic social grouping behaviors of pedestrians," IEEE Journal of Selected Topics in Signal Processing, vol. 9, no. 2, pp. 317-329, 2015.

[38] I. Achour, T. Bejaoui, A. Busson, and S. Tabbane, "SEAD: A simple and efficient adaptive data dissemination protocol in vehicular ad-hoc networks," Wireless Networks, pp. 1-11, 2015.

[39] B. B. Dubey, N. Chauhan, N. Chand, and L. K. Awasthi, "Priority based efficient data scheduling technique for VANETs," Wireless Networks, pp. 1-17, 2015. 
[40] G. J. Suma and R. Lalitha, "Vehicular Ad hoc Networks: A hybrid approach to data dissemination in exigency situations," Wireless Networks, pp. 1-13, 2016.

[41] R. Hussain, S. Kim, and H. Oh, "Traffic information dissemination system: Extending cooperative awareness among smart vehicles with only singlehop beacons in VANET," Wireless Personal Communications, vol. 88, no. 2, pp. 151-172, 2016.

[42] J. Wang, C. Jiang, L. Gao, S. Yu, Z. Han, and Y. Ren, "Complex network theoretical analysis on information dissemination over vehicular networks," in Communications (ICC), 2016 IEEE International Conference on, 2016, pp. 1-6: IEEE.

[43] Z. Amjad, W. C. Song, K. J. Ahn, and M. Shoaib, "Context aware data aggregation in vehicular ad-hoc networks," in Network Operations and Management Symposium (NOMS), 2016 IEEE/IFIP, 2016, pp. 12571260: IEEE.

[44] A. S. K. Mammu, J. Jiru, and U. H. Jayo, "Cluster based semantic data aggregation in VANETs," in 2015 IEEE 29th International Conference on Advanced Information Networking and Applications, 2015, pp. 747-753: IEEE.

[45] C. Ji, Z. Mi, W. Wang, G. Chen, and J. Yang, "A costawareness multicast approach for vehicular ad hoc networks," in Consumer Electronics-Taiwan (ICCE-TW), 2016 IEEE International Conference on, 2016, pp. 1-2: IEEE.

[46] O. Rehman, M. Ould-Khaoua, and H. Bourdoucen, "An adaptive relay nodes selection scheme for multi-hop broadcast in VANETs," Computer Communications, vol. 87, pp. 76-90, 8/1/ 2016.

[47] A. M. Vegni and V. Loscri, "A survey on vehicular social networks," IEEE Communications Surveys \& Tutorials, vol. 17, no. 4, pp. 2397-2419, 2015.

[48] A. M. Vegni, V. Loscrì, and A. V. Vasilakos, Vehicular Social Networks. CRC Press, 2017.

[49] M. Fathian, G. R. Shiran, and A. R. JafarianMoghaddam, "Two New Clustering Algorithms for Vehicular Ad-Hoc Network Based on Ant Colony System," Wireless Personal Communications, vol. 83, no. 1, pp. 473-491, 2015.

[50] R. Goonewardene, F. Ali, and E. Stipidis, "Robust mobility adaptive clustering scheme with support for geographic routing for vehicular ad hoc networks," IET Intelligent Transport Systems, vol. 3, no. 2, pp. 148-158, 2009.

[51] L. Bononi and M. Di Felice, "A cross layered mac and clustering scheme for efficient broadcast in vanets," in Mobile Adhoc and Sensor Systems, 2007. MASS 2007. IEEE International Conference on, 2007, pp. 1-8: IEEE.

[52] R. Aquino and A. Edwards, "A reactive location routing algorithm with cluster-based flooding for inter-vehicle communication," Computación ySistemas, vol. 9, no. 4, pp. 297-313, 2006.

[53] A. Singh and S. Kad, "A secure clustering based approach in vehicular adhoc network," in Signal
Processing, Communication, Power and Embedded System (SCOPES), 2016 International Conference on, 2016, pp. 1127-1133: IEEE.

[54] S. A. Mohammad and C. W. Michele, "Using traffic flow for cluster formation in vehicular ad-hoc networks," in Local Computer Networks (LCN), 2010 IEEE 35th Conference on, 2010, pp. 631-636: IEEE.

[55] V. Vèque, F. Kaisser, C. Johnen, and A. Busson, "CONVOY: A New Cluster-Based Routing Protocol for Vehicular Networks," Vehicular Networks, pp. 91-129, 2013.

[56] B. Ducourthial, Y. Khaled, and M. Shawky, "Conditional transmissions: Performance study of a new communication strategy in VANET," IEEE Transactions on Vehicular Technology, vol. 56, no. 6, pp. 3348-3357, 2007.

[57] U. M. Colesanti, C. Crociani, and A. Vitaletti, "On the accuracy of OMNeT in the Wireless Sensor Networks domain: Simulation vs. testbed," ed: Association for Computing Machinery, 2007, pp. 25-31.

[58] M. A. Mahdi and S. T. Hasson, "A Contribution to the Role of the Wireless Sensors in the IoT Era," Journal of Telecommunication, Electronic and Computer Engineering, vol. 9, no. 2-11, pp. 1-6, 20172017.

[59] B. Musznicki and P. Zwierzykowski, "Survey of simulators for wireless sensor networks," International Journal of Grid and Distributed Computing, vol. 5, no. 3, pp. 23-50, 2012.

[60] J. Helkey, L. Holder, and B. Shirazi, "Comparison of simulators for assessing the ability to sustain wireless sensor networks using dynamic network reconfiguration," Sustainable Computing: Informatics and Systems, vol. 9, pp. 1-7, 2016.

[61] S. Wan, Q. Cao, and D. Wang, "Aoc based vehicular Adhoc network modeling research," in Computer Science and Network Technology (ICCSNT), 2012 2nd International Conference on, 2012, pp. 964-967: IEEE.

[62] U. Wilensky and W. Rand, An introduction to agentbased modeling: modeling natural, social, and engineered complex systems with NetLogo. MIT Press, 2015.

[63] S. Glass, I. Mahgoub, and M. Rathod, "Rapid prototyping of cooperative caching in a VANET: A case study," in Wireless Days, 2017, 2017, pp. 201-204: IEEE.

[64] S. T. Hasson and Z. Y. Hasan, "Roads clustering approach's in VANET models," in New Trends in Information \& Communications Technology Applications (NTICT), 2017 Annual Conference on, 2017, pp. 316321: IEEE.

[65] W. Dongliang and C. Qing, "Multi-agent Based Modeling Simulation about VANET," 2013.

[66] GraphStream. (2017, 30th March ). Available: http://graphstreamproject.org/ 